\title{
Impact of Perioperative Management for Intracranial Arterial Stenosis on Postoperative Stroke Incidence in Coronary Artery Bypass Surgery: A Propensity Matching and Multivariate Analyses
}

\author{
Hajime Imura1*, Yuji Maruyama1, Makoto Shirakawa1, Takashi Nitta2 \\ ${ }^{1}$ Department of Cardiovascular Surgery, Nippon Medical School Musashikosugi Hospital, Kawasaki, Japan \\ ${ }^{2}$ Nippon Medical School Hospital, Tokyo, Japan

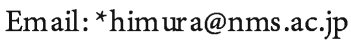

How to cite this paper: Imura, H., Maruyama, Y., Shirakawa, M. and Nitta, T. (2018) Impact of Perioperative Management for Intracranial Arterial Stenosis on Postoperative Stroke Incidence in Coronary Artery Bypass Surgery: A Propensity Matching and Multivariate Analyses. Surgical Science, 9, 311-324.

https://doi.org/10.4236/ss.2018.99038

Received: August 6, 2018

Accepted: September 25, 2018

Published: September 28, 2018

Copyright $(9) 2018$ by authors and Scientific Research Publishing Inc. This work is licensed under the Creative Commons Attribution International License (CC BY 4.0).

http://creativecommons.org/licenses/by/4.0/

\begin{abstract}
Background: Intracranial arterial steno-occlusive lesion (IAS) is the most common cause of stroke. We hypothesized that adequate perioperative management for IAS is useful to prevent stroke in coronary artery bypass grafting (CABG). Methods: IAS was evaluated using magnetic resonance imaging/angiography (MRI/A, $\mathrm{n}=216$ ) in patients without a history of stroke or carotid stenosis $(\geq 50 \%)$ in CABG between May 2005 and June $2018(\mathrm{n}=424)$. Off-pump CABG was performed in most patients. Different perioperative managements of systolic arterial pressure (SAP) were applied for patients with and without MRI/A. SAP was strictly maintained at $\geq 80 \mathrm{mmHg}$ in patients with significant IAS $(\geq 50 \%, \mathrm{n}=28)$ and $\geq 90 \mathrm{mmHg}$ for severe IAS ( $\geq 70 \%, \mathrm{n}=44$ ) while SAP less than $80 \mathrm{mmHg}$ (usually $\geq 70 \mathrm{mmHg}$ ) was allowed for patients without MRI/A. Intra-aortic balloon pumping was used for patients with multiple severe IAS and/or occlusion. Clinical outcomes were compared between patients with and without MRI/A. Propensity matching was performed ( $\mathrm{n}=181$ each). Results: Complete revascularization was achieved in all patients. Nine patients $(2.12 \%)$ had postoperative strokes; the incidence of stroke was significantly less in patients with preoperative MRI/A ( $\mathrm{n}=1, p=0.016)$. There was no significant difference in the incidence of in-hospital mortality between patients with and without preoperative MRI/A $(0.5 \%$ and $1.0 \%$, respectively; $p=0.529)$. Multivariate analysis revealed that no preoperative MRI/A was the only significant predictors of postoperative stroke $(p=0.045)$. The incidence of stroke was not higher in patients with
\end{abstract}


IAS who underwent MRI/A $(p=0.332)$. Conclusions: Preoperative evaluation of IAS using MRI/A and following our perioperative management significantly reduced perioperative stroke in patients undergoing CABG.

\section{Keywords}

CABG, Intracranial Arterial Stenosis, Stroke, Magnetic Resonance Imaging, Off-Pump

\section{Introduction}

Cerebrovascular disease (CVD) is an important risk factor for perioperative stroke in patients undergoing coronary artery bypass grafting (CABG) [1] [2]. Although numerous studies have investigated this critical issue and extracranial carotid disease has been focused to solve this problem, the incidence of stroke has not been reduced in recent decades [3]. This unsatisfactory situation suggests the need for a different approach to this problem.

In general, intracranial arterial steno-occlusive lesion (IAS) is known as the most common cause of stroke [4]. Previous studies demonstrated that the prevalence of IAS was equal to or even higher than that of extracranial carotid lesions in patients undergoing $C A B G$ and that its severity was parallel to the number of diseased coronary arteries [5]. A large-scale study showed that total assessment of CVD using magnetic resonance imaging and angiography (MRI/A) was the most useful method to predict perioperative stroke in patients undergoing CABG [6]. Nevertheless, IAS is not routinely evaluated preoperatively in many patients undergoing CABG.

Previously, we reported that high intraoperative arterial pressure was a fundamental strategy in CABG for patients with severe CVD [7], which is supported by many studies [8] [9]. Schoof et al. observed a high rate of stroke (3 of 11 patients) in standard CABG for patients with reduced cerebrovascular reserve [10]. Contrary to their finding, we could successfully prevent stroke in 43 consecutive patients with chronic brain hypoperfusion (mainly associated with impaired cerebrovascular reserve) by adopting off-pump CABG and keeping arterial pressure high during CABG [11]. Therefore, the substantial circumstantial evidence demonstrates the efficacy of high arterial pressure for patients with CVD; however, only limited studies have compared perioperative stroke rates between patients with and those without the management of arterial pressure in the perioperative period of CABG. Furthermore, there have been also few reports which showed the importance of preoperative evaluation and perioperative management of IAS for patients undergoing CABG.

In the present study, we focused on patients without significant $(\geq 50 \%)$ stenosis on extracranial carotid and vertebral arteries in carotidecho, and evaluated IAS using MRI/A before CABG. High arterial pressure was strictly maintained for patients with IAS and clinical outcomes were compared between patients 
with and without preoperative evaluation and perioperative management of IAS. Propensity score matching was used to reduce the bias between patients with and without preoperative MRI/A. The aim of this study was to investigate the efficacy of our evaluation and management of IAS in preventing perioperative stroke in CABG.

\section{Patients and Methods}

This study was approved by our institutional ethics committee, which judged that no written consent was required for the study (a waiver of consent was obtained). Five hundred eighty consecutive patients underwent carotid echography prior to isolated CABG between January 2005 and June 2018. Patients who had significant carotid stenosis ( $\geq 50 \%$ ), neurological symptoms, or previous symptomatic stroke were excluded. Patients who were unable to undergo MRI (emergency patients, patients with infusion pump, patients under support of a respirator and so on) were also excluded. A total of 424 patients were finally recruited to the study. We first determined the date of CABG from the patients and attending cardiologists (usually between 2 and 4 weeks after coronary angiography) and then planned MRI/A of the brain and neck before CABG. Because we occasionally had difficulty performing MRI/A in those who were scheduled to have CABG within a month, 206 patients could not undergo MRI/A whereas 218 patients did undergo MRI/A. We prospectively evaluated and treated these patients as described below.

\subsection{Cerebrovascular Assessment}

Cerebrovascular atherosclerosis was assessed from MRI/A of the brain and as previously described [7] [11] Stenosis of $\geq 50 \%$ of the intracranial carotid, vertebral, and basilar arteries was defined as significant and $\geq 70 \%$ was considered as severe. Lesions of the anterior, middle, and posterior cerebral arteries and the Willis circle were also assessed in accordance with the methods of previous reports [5] [7]. Unilateral vertebral disease was not identified as severe CVD because of its anatomical features.

\subsection{Surgical Strategy and Intraoperative Management}

Off-pump CABG was initially adopted for all patients. Different strategies of management of systolic arterial pressure (SAP) during surgery were applied for patients with and without preoperative MRI/A. The protocol for patients with preoperative MRI/A was based on our previous study [7]. In brief, for patients with significant IAS in MRI/A, SAP was strictly kept at $\geq 80 \mathrm{mmHg}$ even during distal anastomosis and at $\geq 90 \mathrm{mmHg}$ for patients with severe IAS. When SAP could not be maintained at those levels even with noradrenaline (usually up to $0.15 \mu \mathrm{g} / \mathrm{kg} / \mathrm{h}$ minute), intra-aortic balloon pumping (IABP) was used because IABP raises mean arterial pressure by about $10 \mathrm{mmHg}$ in our experience [7] [11]. For patients with more severe IAS (with flow defect suggesting total occlu- 
sion or multiple severe stenoses in the same carotid or vertebral system), IABP was inserted preoperatively (approximately $2 \mathrm{~h}$ before operation) and SAP was kept at $\geq 90 \mathrm{mmHg}$ with IABP support at the ratio of 1:1 during CABG. If IABP was not applicable for these patients, SAP was kept at $\geq 100 \mathrm{mmHg}$. In patients with severe IAS and reduced collateral flow, thiamylal sodium was used to protect the brain during surgery. In patients without marked IAS or preoperative MRI/A, the lowest SAP of approximately $70 \mathrm{mmHg}$ was allowed during distal anastomosis. These targeted lowest SAPs were determined from the relationship between mean arterial pressure and SAP in our previous work [7].

Cardiopulmonary bypass was used if the targeted SAP could not be maintained even with inotropic and IABP supports and CABG was carried out under the beating heart condition. Patients who had severe stenosis $(\geq 75 \%)$ in the main trunk of the left coronary artery and unstable myocardial ischemia and/or hemodynamic status even under sufficient medical treatment were also indicated for IABP [12]. Cardiac wall motion was carefully observed via transesophageal echography in all patients. Positive inotropic agents were used when necessary. Landiolol was given to prevent tachycardia and atrial fibrillation. The ascending aorta was carefully evaluated by preoperative computed tomography and intraoperative echo examination.

\subsection{Postoperative Management and Brain MRI in the Follow-Up Period}

Managements of SAP were continued as long as monitoring of radial blood pressure was carried out, usually for 3 days, in the intensive care unit (ICU). After the patient became conscious and was weaned from the respirator, neurological symptoms were carefully observed. Other postoperative management was carried out in the same fashion as ordinary CABG in our institutes. Antiplatelet drugs were restarted the day following surgery. Beta-blockade was also administered to prevent atrial fibrillation. Neurological events were carefully and prospectively observed throughout the postoperative course.

\subsection{Statistical Analyses}

All statistical analyses were performed with SPSS $25.0 \mathrm{~J}$ for Windows (SPSS Japan, Tokyo, Japan). The primary endpoint was stroke within 30 days after CABG or hospital stay (if the stay $>30$ days). The incidence of death from any cause was also compared between the two groups. Differences between the groups were evaluated by the Mann-Whitney $U$ test for outcomes with continuous numbers or Fisher's exact test for binary outcomes.

In comparisons between patients with and without preoperative MRI/A, propensity score matching was used to reduce the selection bias of applying preoperative MRI/A. In this procedure, the predicted probability of preoperative MRI/A was calculated by fitting a logistic regression model using clinical variables shown in Table 1 (except serum creatinine level). The pairs of patients 
Table 1. Patient characteristics.

\begin{tabular}{|c|c|c|c|}
\hline Characteristic & $\mathrm{MRI} / \mathrm{A}_{\mathrm{a}}(+), \mathrm{n}=218$ & MRI/A (-), n = 206 & $p$ value \\
\hline Age (yr) & $69.8 \pm 9.1$ & $69.7 \pm 9.9$ & 0.887 \\
\hline Octogenarian (\%) & 12.4 & 14.1 & 0.668 \\
\hline Male (\%) & 80.3 & 75.7 & 0.291 \\
\hline Diabetes mellitus (\%) & 55.0 & 56.8 & 0.769 \\
\hline Hypertension (\%) & 78.9 & 84.5 & 0.168 \\
\hline Hyperlipidemia (\%) & 60.0 & 60.7 & 0.921 \\
\hline Hemodialysis (\%) & 10.1 & 7.8 & 0.497 \\
\hline Serum creatinine $_{\mathrm{b}}(\mathrm{mg} / \mathrm{dl})$ & $0.99 \pm 0.37$ & $1.00 \pm 0.51$ & 0.194 \\
\hline Peripheral arterial disease (\%) & 18.3 & 13.1 & 0.145 \\
\hline 3-vessel disease (\%) & 74.8 & 75.8 & 0.823 \\
\hline No. of $\mathrm{CAD}_{\mathrm{c}}$ & $2.7 \pm 0.5$ & $2.7 \pm 0.6$ & 0.909 \\
\hline $\operatorname{LMT}(\%)_{\mathrm{d}}$ & 25.7 & 31.6 & 0.197 \\
\hline LVEF $<40 \%(\%)$ & 16.5 & 12.6 & 0.273 \\
\hline
\end{tabular}

a: Magnetic resonance imaging and angiography. b: Patients on hemodialysis were excluded from the calculation of serum creatinine. c: Number of diseased coronary arteries. d: Left main trunk stenosis $\geq 50 \%$.

with and without preoperative MRI/A were derived using 1:1 nearest-neighbor matching with a \pm 0.01 caliper and no replacement. A standardized mean difference greater than 0.1 was considered to indicate imbalance. Univariate and multivariate logistic regression analyses were also performed to investigate the risk factors for stroke. A $p$ value of $<0.05$ was considered to indicate statistical significance in all analyses.

\section{Results}

\subsection{Preoperative Assessments}

Table 1 shows that patients in this study were relatively older and reported with more diabetes, hypertension, and three-vessel disease than those in previous studies. Thus, general risk factors for systemic atherosclerosis were common among the patients in this study (Table 1). In comparison between patients with and without preoperative MRI/A, hypertension, peripheral arterial disease, left main disease and low ejection fraction seemed to be more common in patients with preoperative MRI/A; however, after propensity score matching, these two groups were well balanced in all baseline characteristics (Table 2).

\subsection{Atherosclerotic Changes on MRI/A}

The distribution of IAS is shown in Figure 1, and representative MRA images are shown in Figure 2. Significant stenosis was observed in 72 patients (33.0\%) and severe IAS was in 44 patients (20.2\%) on MRI/A. Eight patients (18.2\%) showed severe bilateral vertebral and/or basilar arterial disease without significant 
Table 2. Baseline characteristics of propensity-matched patients.

\begin{tabular}{ccccc}
\hline Characteristic & MRI/A $\mathrm{A}_{\mathrm{a}}+($ & $\mathrm{MRI} / \mathrm{A}_{\mathrm{a}}(-)$ & $p$ value & $\mathrm{SMD}_{\mathrm{b}}$ \\
\hline No. of patients & 181 & 181 & & \\
Age (yr) & $70.0 \pm 9.1$ & $69.8 \pm 9.8$ & 0.841 & 0.021 \\
Octogenarian (\%) & 13.3 & 12.2 & 0.875 & -0.030 \\
Male (\%) & 81.2 & 77.3 & 0.437 & -0.098 \\
Hypertension (\%) & 81.2 & 82.3 & 0.892 & -0.026 \\
Diabetes (\%) & 55.8 & 54.1 & 0.833 & 0.040 \\
Hyperlipidemia (\%) & 61.3 & 60.8 & 1.000 & 0.000 \\
Hemodialysis (\%) & 8.3 & 8.3 & 1.000 & 0.000 \\
PAD (\%) & 17.1 & 14.9 & 0.668 & 0.054 \\
No. of CAD (\%) & $2.72 \pm 0.57$ & $2.69 \pm 0.54$ & 0.332 & -0.054 \\
LMT (\%) & 27.6 & 28.7 & 0.908 & -0.022 \\
LVEF < 40\% (\%) & 12.2 & 14.4 & 0.642 & -0.059 \\
Serum creatinine & & $0.99 \pm 0.35$ & 0.356 & -0.067 \\
EuroSCORE & $6.48 \pm 8.45$ & $6.69 \pm 6.53$ & 0.462 & 0.029 \\
\hline
\end{tabular}

a: Magnetic resonance imaging and angiography. b: Standardized mean difference. c: Peripheral artery disease. d: Number of diseased coronary arteries. e: Left main trunk stenosis $\geq 50 \%$. f: Patients with hemodialysis were excluded from the calculation of serum creatinine.

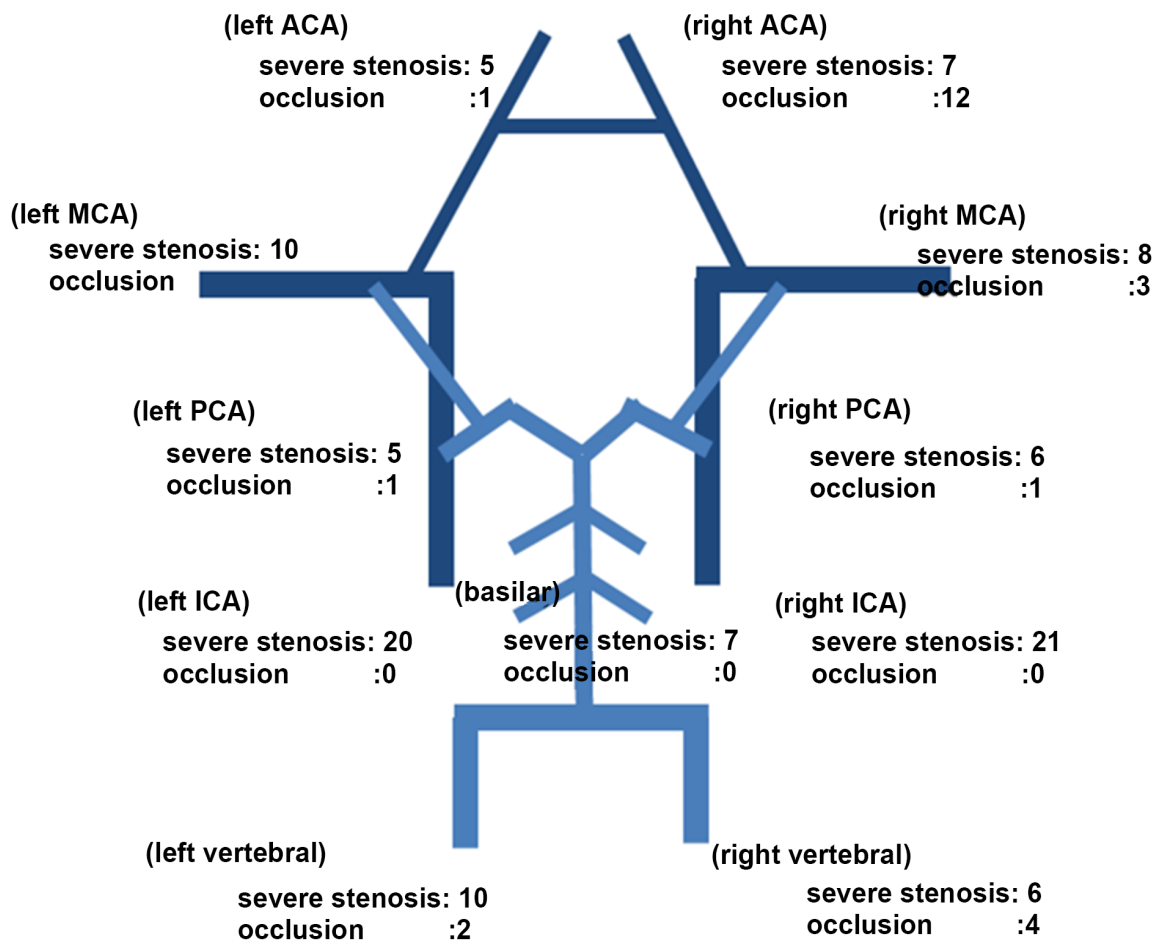

Figure 1. Distribution of intracranial arterial stenosis $(\geq 70 \%)$ and occlusion onmagnetic resonance angiography (MRA). 


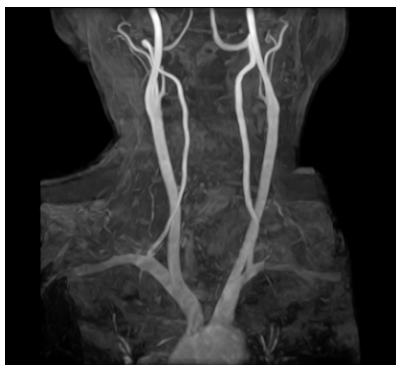

(a)

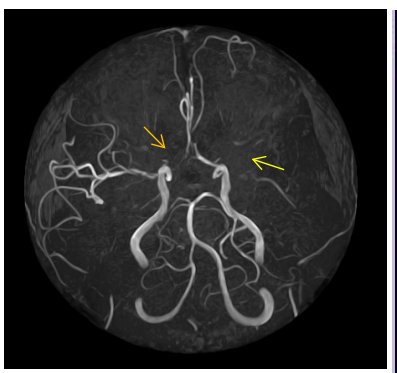

(b)

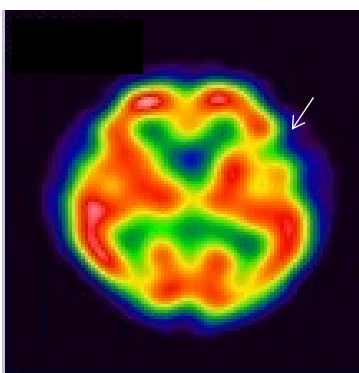

(c)

(A)

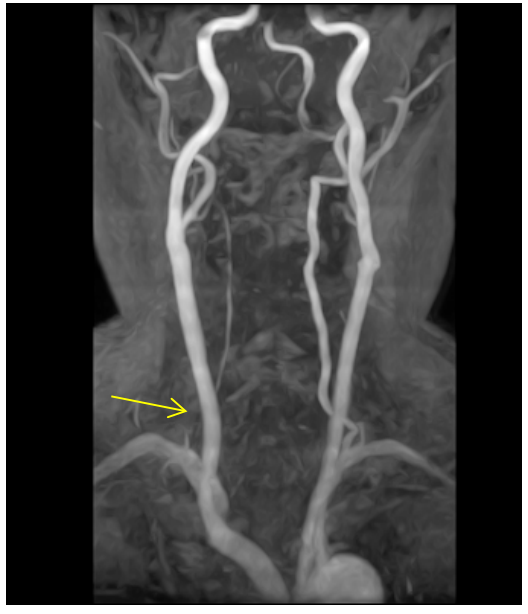

(a)

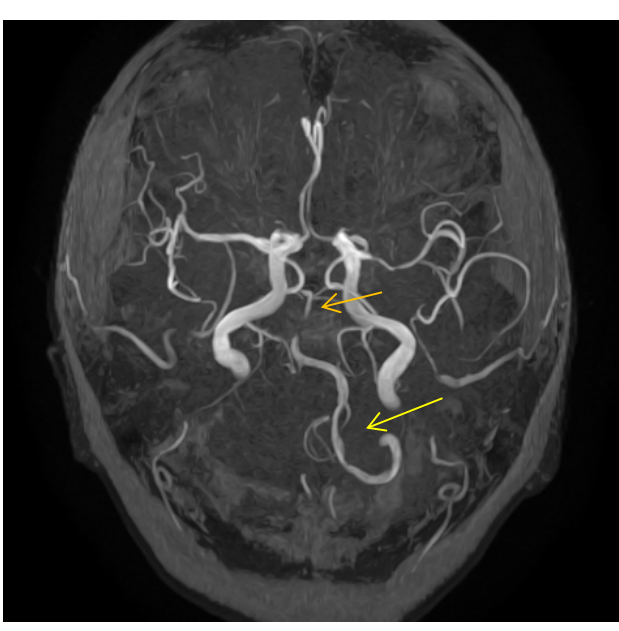

(b)

(B)

Figure 2. (A) There is no significant atherosclerotic lesion in magnetic resonance angiography (MRA) of the neck (a), while severe steno-occlusive lesions are seen in intracranial arteries (b). Yellow arrow shows occlusion of the left middle cerebral artery and orange arrow indicates severe stenosis of the right anterior cerebral artery. Hypoperfusion is seen (white arrow) in an area of the left middle cerebral artery (c). (B) The right vertebral arterywas occluded (yellow arrow) in the neck MRA (a). This was also seen while stenosis $(\geq 50 \%)$ of the left vertebral artery was not observed in echo examination. In the brain MRA, stenosis of the left vertebralartery was severe (yellow arrow) and basilar artery was occluded (orange arrow, b).

IAS in the carotid system. Thus, silent IAS without a history of neurological symptoms and extracranial carotid disease was not rare in this study.

\subsection{Clinical Outcomes}

Intraoperative parameters and clinical outcomes are shown in Table 3. Complete revascularization was achieved in all patients. The mean numbers of distal anastomoses were 3.8 and 3.9 for patients with and without MRI/A, respectively $(p=0.454)$; approximately half of them were without vein graft in both groups. IABP was used to maintain sufficient SAP for the brain in seven patients with severe IAS, but there was no significant difference in frequency of IABP use between patients with and without MRI/A. Cardiopulmonary bypass was used for 
Table 3. Intraoperative and postoperative parameters.

\begin{tabular}{cccc}
\hline Parameters & MRI/A $_{\mathrm{a}}(+), \mathrm{n}=218$ & MRI/A $(-), \mathrm{n}=206$ & $p$ value \\
\hline Intraoperative & & & \\
Graft (n) & $3.8 \pm 1.4$ & $3.9 \pm 1.3$ & 0.454 \\
Use of ITA (\%) & 96.8 & 98.1 & 0.340 \\
All arterial grafts (\%) & 52.3 & 47.1 & 0.287 \\
Use of IABP (\%) & 21.6 & 19.9 & 0.720 \\
Use of pump (\%) & 4.1 & 6.8 & 0.285 \\
Postoperative & & & \\
Atrial fibrillation (\%) & 23.9 & 20.4 & 0.414 \\
Ventilation time (h) & $13.8 \pm 15.2$ & $15.8 \pm 12.7$ & 0.210 \\
ICU stay (days) & $3.2 \pm 1.9$ & $3.4 \pm 1.9$ & 0.421 \\
Hospital stay (days) & $19.5 \pm 10.9$ & $21.0 \pm 15.5$ & 0.882 \\
Stroke (\%) & 0.5 & 3.9 & 0.016 \\
Hospital death (\%) & 0.5 & 1.0 & 0.614 \\
Graft patency (\%) & 98.8 & 98.1 & 0.136 \\
\hline
\end{tabular}

a: Magnetic resonance imaging and angiography. b: Internal thoracic artery. c: Intra-aortic balloon pumping. d: Graft patency was generally examined within a month after surgery by angiography or computed tomography.

20 patients, and all anastomoses were performed on the beating heart with sufficient SAP. The postoperative course was similar in the two groups regarding the ventilation time, ICU stay, and hospital stay; however, the incidence of stroke was significantly lower in patients with MRI/A than those without MRI/A ( $p=$ 0.016). These findings on intra- and postoperative parameters were similar despite propensity score matching (Table 4).

\subsection{Postoperative Stroke}

Nine patients (2.12\%) had ischemic strokes in the postoperative period, and one patient died due to stroke (Table 5). All strokes were diagnosed with MRI/A, and six of nine strokes occurred in the middle cerebral artery area. One stroke occurred in a patient with preoperative MRI/A, and eight strokes occurred in patients without preoperative MRI/A $(p=0.016)$. In the patient with preoperative MRI/A, severe IAS was recognized on the MRI/A, and therefore SAP was strictly maintained at or higher than $100 \mathrm{mmHg}$ throughout the surgery, but nevertheless an early stroke occurred. Of the nine patients with stroke, we observed severe or significant IAS in seven (six were identified in postoperative MRI/A), and all strokes occurred in the distal areas of the IAS. The incidence of stroke was significantly lower in patients with preoperative MRI/A than in those without preoperative MRI/A in both the unadjusted and the adjusted cohorts (Table 3 and Table 4). Among patients with preoperative MRI/A, there was no significant difference in the incidence of stroke between patients with and 
Table 4. Intraoperative and postoperative parameters in matched patients.

\begin{tabular}{cccc}
\hline Parameters & MRI/A (+) & MRI/A $\mathrm{A}_{\mathrm{a}}(-)$ & $p$ value \\
\hline Intraoperative & & & 0.268 \\
Graft (n) & $3.76 \pm 1.373$ & $3.93 \pm 1.319$ & 0.502 \\
Use of ITA (\%) b $_{\mathrm{b}}$ & 98.3 & 96.9 & 0.527 \\
All arterial grafts (\%) & 43.6 & 47.5 & 0.502 \\
Use of IABP (\%) & 17.0 & 21.0 & 0.654 \\
Use of pump (\%) & 6.6 & 5.0 & 0.527 \\
Postoperative & & 23.8 & 0.143 \\
Atrial fibrillation (\%) & 20.4 & $14.2 \pm 15.8$ & 0.393 \\
Ventilation time (h) & $14.9 \pm 12.3$ & $3.2 \pm 1.8$ & 0.794 \\
ICU stay (days) & $3.4 \pm 2.0$ & $20.1 \pm 11.5$ & 0.037 \\
Hospital stay (days) & $21.5 \pm 16.2$ & 4.4 & 1.000 \\
Stroke (\%) & 0.6 & 0.6 & 0.225 \\
Hospital death (\%) & 0.6 & 99.0 & \\
Graft patency (\%) & 98.4 & & \\
\hline
\end{tabular}

a: Magnetic resonance imaging and angiography. b: Internal thoracic artery. c: Intra-aortic balloon pumping. d: Graft patency was generally examined within a month after surgery by angiography or computed tomography.

Table 5. Details of stroke cases.

\begin{tabular}{|c|c|c|c|c|c|c|}
\hline No. & Type & Age (yr) & Preoperative $\mathrm{MRA}_{\mathrm{a}}$ & Stroke focus & Details of $\mathrm{CVD}_{\mathrm{b}}$ & Consequence at discharge \\
\hline 1 & Late and embolic & 73 & Not performed & Left $\mathrm{MCA}_{\mathrm{c}}$ area & $\begin{array}{l}\text { Irregularity of bilateral } \\
\qquad \mathrm{ICA}_{\mathrm{d}} \text { and } \mathrm{MCA}\end{array}$ & $\begin{array}{l}\text { Alive, mild right } \\
\text { hemiparesis }\end{array}$ \\
\hline 2 & Early and embolic & 88 & Not performed & Left MCA area & $\begin{array}{l}\text { Severe stenosis } \\
\text { of left MCA }\end{array}$ & $\begin{array}{l}\text { Alive, mild right } \\
\text { hemiparesis }\end{array}$ \\
\hline 3 & $\begin{array}{l}\text { Late and hypo } \\
\text { perfusion }\end{array}$ & 65 & Not performed & Right $\mathrm{PCA}_{\mathrm{e}}$ area & $\begin{array}{l}\text { Severe stenosis of bilateral } \\
\mathrm{VA}_{\mathrm{f}} \text {, basilar and PCA }\end{array}$ & Alive,vision disorder \\
\hline 4 & $\begin{array}{c}\text { Late and } \\
\text { hypoperfusion }\end{array}$ & 79 & Not performed & Right MCA area & $\begin{array}{l}\text { Severe stenosis of bilateral } \\
\text { ICA and right MCA }\end{array}$ & Alive, left hemiparesis \\
\hline 5 & Late and embolic & 72 & Not performed & Left MCA area & $\begin{array}{c}\text { Severe stenosis of left ICA } \\
\text { and MCA }\end{array}$ & $\begin{array}{l}\text { Died, unconsciousness } \\
\text { and right hemiplegia }\end{array}$ \\
\hline 6 & $\begin{array}{c}\text { Early and } \\
\text { hypoperfusion }\end{array}$ & 79 & Not performed & Whole brain & None & Alive, unconsciousness \\
\hline 7 & Late and embolic & 79 & Not performed & $\begin{array}{c}\text { Left } \mathrm{ACA}_{\mathrm{g}} \text { area, } \\
\text { mesencephalon and } \\
\text { cerebellum }\end{array}$ & None & Alive, diplopia \\
\hline 8 & Early and embolic & 69 & Not performed & Left MCA area & $\begin{array}{c}\text { Irregularity of left ICA } \\
\text { and MCA }\end{array}$ & $\begin{array}{l}\text { Alive, numbness of left } \\
\text { upper extremity }\end{array}$ \\
\hline 9 & Early and embolic & 80 & Severe CVD & Right MCA area & $\begin{array}{l}\text { Severe stenosis of right } \\
\text { ICA and MCA }\end{array}$ & Alive, mild left hemiparesis \\
\hline
\end{tabular}

a: Magnetic resonance imaging and angiography. b: Cerebrovascular disease. c: Middle cerebral artery. d: Internal carotid artery. e: Posterior cerebral artery. f: Vertebral artery. g: Anterior cerebral artery.

without IAS $(p=0.332)$. Multivariate analysis revealed that absence of preoperative MRI/A was the significant factors for postoperative stroke (Table 6). 
Table 6. Analysis of risk factors for stroke.

\begin{tabular}{|c|c|c|c|}
\hline Variable & $p$ value & Hazard ratio & $95 \% \mathrm{CI}_{\mathrm{a}}$ \\
\hline \multicolumn{4}{|l|}{ Univariate analysis } \\
\hline Age & 0.046 & 1.096 & $1.001-1.199$ \\
\hline Octogenarian & 0.427 & 1.910 & $0.387-9.435$ \\
\hline Male & 0.983 & 0.983 & $0.201-4.814$ \\
\hline Hypertension & 0.245 & 0.434 & $0.106-1.775$ \\
\hline Diabetes & 0.983 & 0.986 & $0.261-3.724$ \\
\hline Hyperlipidemia & 0.765 & 0.817 & $0.216-3.086$ \\
\hline Hemodialysis & 0.998 & 0.000 & 0.000 \\
\hline Peripheral arterial disease & 0.699 & 0.661 & $0.081-5.373$ \\
\hline No. of $\mathrm{CADs}_{\mathrm{b}}$ & 0.692 & 1.318 & $0.336-5.166$ \\
\hline $\mathrm{LMT}_{\mathrm{c}}$ & 0.694 & 0.728 & $0.149-3.554$ \\
\hline LVEF $<40 \%$ & 0.762 & 0.723 & $0.089-5.887$ \\
\hline Preoperative MRI/A $\mathrm{d}_{\mathrm{d}}$ & 0.042 & 0.114 & $0.014-0.920$ \\
\hline EuroSCORE & 0.205 & 1.039 & $0.979-1.102$ \\
\hline No. of distal anastomosis & 0.268 & 1.330 & $0.805-2.177$ \\
\hline Aortic no-touch & 0.488 & 0.610 & $0.150-2.470$ \\
\hline Use of $\mathrm{CPB}_{e}$ & 0.998 & 0.000 & 0.000 \\
\hline $\mathrm{POAF}_{\mathrm{f}}$ & 0.421 & 1.780 & $0.437-7.257$ \\
\hline \multicolumn{4}{|l|}{ Multivariate analysis } \\
\hline Age & 0.052 & 1.088 & $0.999-1.185$ \\
\hline Preoperative MRI/A & 0.045 & 0.117 & $0.014-0.951$ \\
\hline
\end{tabular}

a: Confidence interval. b: Number of diseased coronary arteries. c: Left main trunk stenosis $\geq 50 \%$. d: Magnetic resonance imaging/angiography. e: Cardiopulmonary bypass. f: Postoperative atrial fibrillation.

\subsection{In-Hospital Mortality}

Three patients (0.7\%) died in the hospital because of sepsis with acute respiratory distress syndrome, cardiac and renal failure, and stroke. In-hospital mortality was significantly higher in patients with postoperative stroke than in those without postoperative stroke $(p=0.002)$.

\section{Discussion}

The present study focused on patients without a stroke history and extracranial CVD and revealed that patients who underwent preoperative evaluation of IAS and received adequate perioperative management for IAS had a lower incidence of perioperative stroke than patients without those procedures in CABG. Although this study was not a randomized trial, propensity score matching successfully reduced the selection bias between patients with and without preoperative MRI/A. These two groups had similar perioperative parameters except for 
the incidence of stroke in prospective observation. Therefore, we are convinced of this result. To the best of our knowledge, this is the first study reporting that adequate perioperative management of IAS could decrease the incidence of stroke in CABG.

This study determined a marginally higher incidence of IAS than previous studies [2] [5]. Higher proportions of hypertension, diabetes, and hyperlipidemia in our cohort than in previous studies may explain this high prevalence of IAS [13] [14]. Although these factors are identified as common predictors of IAS and external carotid disease, it is known that IAS and external carotid disease do not have the same prevalence and distribution [2] [5]. In general, IAS is more common than external carotid disease in Asians as well as African and Hispanic, and only $10 \%-20 \%$ of patients with IAS have external carotid disease [5]. On the basis of these observations, screening for IAS (in addition to carotid echography) is recommended in patients at high risk for systemic atherosclerosis and/orcertain races. Previous studies have shown that approximately half or more of strokes occurring after CABG are not explained by carotid disease [3] [15]. The present study indicates that IAS may explain the gap between stroke focuses and carotid diseases.

Although embolism is the most frequent cause of stroke in patients undergoing CABG, a recent guideline referred to the importance of brain hypoperfusion as an underlying mechanism of stroke [16]. This is because hypoperfusion itself is an important cause of embolism by creating prothrombotic conditions and suppressing the ability to wash out emboli in the distal arteries [17]. Because a steno-occlusive lesion close to brain tissue can affect distal perfusion more strongly than a remote lesion like that of the extracranial carotid artery [17], IAS must be given serious attention. Thus, severe IAS can be a mechanism of late stroke due to an embolism caused by atrial fibrillation as well as of intraoperative stroke due to brain hypoperfusion. In the absence of IAS data in patients without preoperative MRI/A, we cannot convincingly state that IAS was a significant risk factor for perioperative stroke, and our strategy was effective in patients with IAS. However, findings of perioperative strokes (seven of nine were IAS-related strokes and all occurred in the distal areas of IAS) strongly suggest the significance of IAS as a risk factor of stroke as well as a target to decrease the incidence of stroke in CABG.

We believe that strict management of SAP is vital to prevent stroke in patients with IAS. Previous study demonstrated that cerebral perfusion was maintained under autoregulatory mechanism when SAP was $\geq 70 \mathrm{mmHg}$ [18]. Another study showed that the risk of acute conversion to on-pump was significantly increased when mean arterial pressure was at $50 \mathrm{mmHg}(\doteqdot 70 \mathrm{mmHg}$ in SAP $)$ or lower in off-pump CABG [19]. These reports suggested that off-pump CABG with SAP at $\geq 70 \mathrm{mmHg}$ should be at an acceptable risk of major complications, however, we observed relatively high stroke incidence with that SAP in patients without preoperative MRI/A. We now think SAP should be strictly 
kept at $\geq 80 \mathrm{mmHg}$ or higher for patients without preoperative IAS evaluation. In general, one-third of perioperative strokes occur intraoperatively, most other strokes occur within the next 3 days, and the incidence becomes constant at a low level after 6 days [20]. Therefore, the strict management of SAP should be continued beyond surgeries. Since the stay in ICU was usually for 3 days in many of patients in this study, we think our strategy was reasonable for patients with IAS.

Serruys et al. observed major adverse cerebrovascular or cardiac events in $0.9 \%$ of patients in the approximately 2 weeks before CABG [14]. Thus, preoperative adverse events are a problem in current CABG, and a delay in CABG due to preoperative examinations can be a serious disadvantage for the patient. This is the reason we could not perform MRI/A in nearly half of the patients, and we abandoned the attempt to randomize patients in this study. Further investigations are necessary to show the value of preoperative evaluation of IAS.

\section{Limitations of the Study}

Several limitations of the present study should be acknowledged. First, as we mentioned above, this was not a randomized trial, and therefore, some unintentional bias could have influenced the result even though we adopted propensity score matching. Second, we did not perform any special examinations to evaluate encephalopathy and dementia after CABG. However, the patients' families and we did not notice any significant changes. Finally, all patients were ethnically Japanese and were treated by a single surgical team, introducing the possibility of ethnic- and surgeon-based biases. However, we believe that our investigation is useful, because more than half of the people in the world are Asian, and the majority are counted in the high-risk IAS (Asian and races of African and Hispanic) group [4].

\section{Conclusion}

In this study, the preoperative evaluation of intracranial atherosclerosis using MRI/A and following our perioperative management for patients with IAS markedly decreased the incidence of perioperative stroke in patients undergoing CABG. Because most strokes occurred in the distal area of IAS, IAS could be a crucial cause of perioperative stroke. Therefore, we recommend preoperative brain MRI/A for patients with high risk of cerebrovascular atherosclerosis in CABG.

\section{Conflicts of Interest}

The authors declare no conflicts of interest regarding the publication of this paper.

\section{References}

[1] Ito, A., Goto, T., Maekawa, K., Baba, T., Mishima, Y. and Ushijima K. (2012) Post- 
operative Neurological Complications and Risk Factors for Pre-Existing Silent Brain Infarction in Elderly Patients Undergoing Coronary Artery Bypass Grafting. Journal of Anesthesia, 26, 405-411. https://doi.org/10.1007/s00540-012-1327-4

[2] Luchowski, P., Wojczal, J., Buraczynska, K., Kozlowicz, M., Stazka, J. and Rejdak, K. (2015) Predictors of Intracranial Cerebral Artery Stenosis in Patients before Cardiac Surgery and Its Impact on Perioperative and Long Term Stroke Risk. Neurologia $i$ Neurochirurgia Polska, 49, 395-400.https://doi.org/10.1016/j.pjnns.2015.09.006

[3] Naylor, A.R. (2009) Does the Risk of Post-CABG Stroke Merit Staged or Synchronous Reconstruction in Patients with Symptomatic or Asymptomatic Carotid Disease? The Journal of Cardiovascular Surgery, 50, 71-81.

[4] Carvalho, M., Oliveira, A., Azevedo, E. and Bastos-Leite, A.J. (2014) Intracranial Arterial Stenosis. Journal of Stroke and Cerebrovascular Disease, 23, 599-609. https://doi.org/10.1016/j.jstrokecerebrovasdis.2013.06.006

[5] Uekita, K., Hasebe, N., Funayama, N., Aoyama, H., Kuroda, K., Aizawa, H., et al. (2003) Cervical and Intracranial Atherosclerosis and Silent Brain Infarction in Japanese Patients with Coronary Artery Disease. Cerebrovascular Diseases, 16, 61-68. https://doi.org/10.1159/000070117

[6] Lee, E.J., Choi, K.H., Ryu, J.S., Jeon, S.B., Lee, S.W., Park, S.W., et al. (2011) Stroke Risk after Coronary Artery Bypass Graft Surgery and Extent of Cerebral Artery Atherosclerosis. Journal of the American College of Cardiology, 57, 1811-1818. https://doi.org/10.1016/j.jacc.2010.12.026

[7] Imura, H., Nishina, D., Maruyama, Y., et al. (2013) Coronary Artery Bypass Surgery for Patients with Severe Cerebrovascular Atherosclerosis: Importance of Preoperative Assessment and Perioperative Management. Surgical Science, 4, 535-542. https://doi.org/10.4236/ss.2013.412104

[8] Gottesmann, R.F. and Wityk, R.J. (2006) Brain Injury from Cardiac Bypass Procedures. Seminars in Neurology, 26, 432-439. https://doi.org/10.1055/s-2006-948324

[9] Imasaka, K., Yasaka, M., Tayama, E. and Tomita, Y. (2015) Obstructive Carotid and/or Intracranial Artery Disease Rarely Affects the Incidence of Haemodynamic Ischemic Stroke During Cardiac Surgery: A Study on Brain Perfusion Single-Photon Emission Computed Tomography with Acetazolamide. European Journal of Cardio-Thoracic Surgery, 48, 739-746. https://doi.org/10.1093/ejcts/ezu502

[10] Schoof, J., Lubahn, W., Baeumer, M., Kross, R., Wallesch, C.W., Kozian, A., et al. (2007) Impaired Cerebral Autoregulation Distal to Carotid Stenosis/Occlusion Is Associated with Increased Risk of Stroke at Cardiac Surgery with Cardiopulmonary Bypass. The Journal of Thoracic and Cardiovascular Surgery, 134, 690-697. https://doi.org/10.1016/j.jtcvs.2007.03.018

[11] Imura, H., Maruyama, Y., Takahashi, K., Nishina, D. and Nitta, T. (2018) Coronary Artery Bypass Surgery for Patients with Brain Hypoperfusiuon. The Journal of Cardiovascular Surgery, 59, 640-647.

[12] Etienne, P.Y., Papadatos, S., Glineur, D., Mairy, Y., El Khoury, E., Noirhomme, P., et al. (2007) Reduced Mortality in High-Risk Coronary Patients Operated off Pump with Preoperative Intraaortic Balloon Counterpulsation. The Annals of Thoracic Surgery, 84, 498-503. https://doi.org/10.1016/j.athoracsur.2007.03.080

[13] Serruys, P.W., Morice, M.C., Kappetein, A.P., Colombo, A., Holmes, D.R., Mack, M.J., et al. (2009) Percutaneous Coronary Intervention versus coronary-Artery Bypass Grafting for Severe Coronary Artery Disease. New England Journal of Medicine, 360, 961-972. https://doi.org/10.1056/NEJMoa0804626

[14] Marui, A., Okabayashi, H., Komiya, T., Tanaka, S., Furukawa, Y., Kita, T., et al. 
(2012) Benefits of Off-Pump Coronary Bypass Grafting in High-Risk Patients. Circulation, 126, S151-S157. https://doi.org/10.1161/CIRCULATIONAHA.111.083873

[15] Venkatachalam, S., Gray, B.H., Mukherjee, D. and Shishehbor, M.H. (2011) Contemporary Management of Concomitant Carotid and Coronary Artery Disease. Heart, 97, 175-180. https://doi.org/10.1136/hrt.2010.203612

[16] Hills, L.D., Smith, P.K., Anderson, J.L., Bittl, J.A., Byrne, J.G., Cigarroa, J.E., et al. (2011) ACCF/AHA Guideline for Coronary Artery Bypass Graft Surgery: A Report of the American College of Cardiology Foundation/American Heart Association Task Force on Practice Guidelines. Circulation, 124, e652-e735. https://doi.org/10.1161/CIR.0b013e31823c074e

[17] Caplan, L.R., Wong, K.S., Gao, S. and Hennerici, M.G. (2006) Is Hypoperfusion an Important Cause of Strokes? If So, How? Cerebrovascular Diseases, 21, 145-153. https://doi.org/10.1159/000090791

[18] Obara, S. and Murakawa, M. (2016) Controversy; Volume Load vs. Vasopressor. In: Asai, T., Ochi, M. and Yokoyama, H., Eds., Off-Pump Coronary Artery Bypass, Springer, Tokyo, 59-66. https://doi.org/10.1007/978-4-431-54986-4_7

[19] Virmani, S. and Tempe, D.K. (2007) Anaesthesia for Off-Pump Coronary Artery Surgery. Annals of Cardiac Anesthesia, 10, 65-71. https://doi.org/10.4103/0971-9784.37931

[20] Tarakji, K.G., Sabik, J.F., Bhudia, S.K., Batizy, L.H. and Blackstone, E.H. (2011) Temporal Onset, Risk Factors, and Outcomes Associated with Stroke after Coronary Artery Bypass Grafting. JAMA, 305, 381-390.

https://doi.org/10.1001/jama.2011.37 